\title{
Endocarditis infecciosa y embolia séptica pulmonar por Pseudomonas aeruginosa en un paciente con VIH/SIDA
}

\author{
Infective endocarditis and pseudomonal septic \\ embolizaton in a patient with HIVIAIDS
}

\author{
Cristian IVÁn García Rincón, MD. ${ }^{(1)}$; AlejandRa Cañas MD. ${ }^{(2)}$; Santiago Salazar MD. ${ }^{(3)}$; \\ Ricardo ARTURo MARTínez García MD. ${ }^{(4)}$
}

\begin{abstract}
Resumen
Se describe el caso de un paciente de sexo masculino, de 43 años de edad, con diagnóstico de infección por el virus de la inmunodeficiencia humana y síndrome de inmunodeficiencia adquirida, no usuario de drogas intravenosas, quien presentó imágenes pulmonares cavitadas compatibles con embolia séptica en el contexto de endocarditis infecciosa de la válvula pulmonar y bacteriemia por Pseudomonas aeruginosa, con respuestas clínica y ecocardiográfica favorables al manejo con antibioticoterapia. La endocarditis infecciosa derecha es responsable de un 5 a $10 \%$ de todos los casos de endocarditis. Aunque puede presentarse en pacientes con marcapaso permanente, catéter venoso central o cardiopatía congénita, esta situación se observa más frecuentemente en adictos a uso de drogas intravenosas. Mientras la válvula tricúspide es el lugar de infección más común, también se puede observar infección de las válvulas pulmonar y de Eustaquio. En el paciente con infección por el virus de la inmunodeficiencia humana, la incidencia de endocarditis ha disminuido en la era de la terapia antirretroviral, pero es más frecuente en aquellos con inmunosupresión avanzada y genera mayor morbilidad y mortalidad a un año, además de mayor riesgo de embolización arterial (entre ellas pulmonar). La incidencia de endocarditis infecciosa por Pseudomonas aeruginosa se ha estimado en alrededor del $5 \%$ en este grupo de pacientes.
\end{abstract}

Palabras clave: endocarditis infecciosa, embolia séptica pulmonar, virus de la inmunodeficiencia humana, síndrome de inmunodeficiencia adquirida, Pseudomonas aeruginosa.

\begin{abstract}
We describe the case of a 43-year-old male, diagnosed with human immunodeficiency virus infection and acquired immunodeficiency syndrome, not a user of intravenous drugs, who presented with lung cavitation consistent with septic embolization in the context of infective endocarditis of the pulmonary valve and Pseudomonas aeruginosa bacteremia, with a positive clinical and echocardiographic response to antibiotic therapy. Right-sided infective endocarditis is responsible for $5-10 \%$ of all cases of endocarditis. Although it can appear in patients with a permanent pacemaker, central venous catheter, or congenital cardiopathy, this situation is more frequently observed in addicts to intravenous drugs. While the tricuspid valve is the most common site of infection, infection of the pulmonary valve and the Eustachian valve can also be observed. In patients with human immunodeficiency virus infection, the incidence of endocarditis has decreased in the era of antiretroviral therapy; it is more common in patients with advanced immunosuppression, and causes more one-year morbidity and mortality, besides a greater risk of arterial embolizaton (pulmonary among others). The incidence of Pseudomonal infective endocarditis has been estimated at about $5 \%$ in this group of patients.
\end{abstract}

Keywords: Infective endocarditis, septic pulmonary embolization, human immunodeficiency virus, acquired immunodeficiency syndrome, Pseudomonas aeruginosa.

\footnotetext{
${ }^{(1)}$ Médico internista. Universidad tecnológica de Pereira. Hospital Pablo Tobón Uribe. Medellín-Colombia

${ }^{(2)}$ Médica Internista y Neumóloga. Profesora asistente, Pontificia Universidad Javeriana. Hospital Universitario San Ignacio, Bogotá-Colombia.

${ }^{(3)}$ Médico Internista yCardiólogo. Clínica Comfamiliar Risaralda, Pereira-Colombia. ${ }^{(4)}$ Médico Internista e Infectólogo. Clínica Comfamiliar Risaralda, Pereira-Colombia. Correspondencia: Cristian Iván García Rincón. Correo electrónico: vesalio21@yahoo. com

Recibido: 28/04/14. Aceptado: 10/06/14.
} 


\section{Introducción}

La endocarditis infecciosa derecha, representa un 5 a $10 \%$ de todos los casos de endocarditis infecciosa. Aunque puede presentarse en pacientes con marcapasos permanentes, desfibriladores cardiacos implantables, catéteres venosos centrales o cardiopatías congénita, se ha descrito con mayor frecuencia en personas con abuso de drogas intravenosas (ISA, del inglés intravenous substance abuse) (1). En el grupo de pacientes infectados con el virus de la inmunodeficiencia humana (VIH), es más alta su prevalencia en adictos a drogas intravenosas y en aquellos con mayor inmunosupresión. Mientras la válvula tricúspide es el lugar de infección más común, también se puede observar infección de las válvulas pulmonar y de Eustaquio. Los organismos predominantes son Staphylococcus aureus (60-90\%) y Pseudomonas aeruginosa; otros organismos gramnegativos, hongos, enterococos y estreptococos, así como infecciones polimicrobianas, se reportanmenos a menudo $(2,3)$.

La endocarditis infecciosa causada por bacilos gramnegativos no HACEK (Hemophilus, Actinobacillus, Cardiobacterium, Eikenellay Kingella) es una enfermedad rara y pobremente caracterizada. La literatura que describe este evento consiste principalmente de varias series de casos pequeñas a partir de brotes en las décadas de los setenta y ochenta en personas con ISA en grandes áreas urbanas, como Detroit, Cleveland y San Francisco. Como resultado de lo anterior, la endocarditis infecciosa debida a bacilos gramnegativos no-HACEK había sido considerada casi exclusiva de los pacientes con ISA. Paralelamente, la endocarditis infecciosa por estos gérmenes se reporta ocasionalmente como nosocomial $(4,5)$.

La embolia pulmonar no trombótica se define como la migración a la circulación pulmonar de diferentes tipos celulares (adipocitos, hematopoyéticos, amnióticos, trofoblásticos o tumorales), bacterias, hongos, material extraño o gas. La embolización séptica pulmonar es un problema raro pero bien reconocido en el contexto de endocarditis infecciosa del lado derecho y tromboflebitis séptica de orígenes como las amígdalas, regiones pélvicas, dentales o yugulares y catéteres venosos centrales infectados (6).
Se presenta el caso de un paciente de sexo masculino, de 43 años de edad, con antecedente de VIH, a quien se le diagnosticó endocarditis infecciosa de corazón derecho y embolización séptica pulmonar, con adecuada respuesta al tratamiento médico dirigido por cultivos.

\section{Caso}

Paciente de sexo masculino, de 43 años de edad, con antecedentes de infección por VIH y síndrome de inmonodeficiencia adquirida (SIDA), diagnosticados varios años atrás, en tratamiento con lamivudina, abacavir, atazanavir, ritonavir, recuento reciente de linfocitos CD4 de 187 y carga viral de 42.319 copias $/ \mathrm{ml}$, sin historia de toxicomanía, con larga estancia hospitalaria por cuadro consuntivo y diarrea multifactorial, expuesto a tratamiento empírico para tuberculosis (TB) e infecciones fúngicas, quien durante su hospitalización requirió la implantación de catéteres venosos centrales en varias oportunidades, presentando varios cuadros infecciosos, tratados con antibióticos de amplio espectro.

A su ingreso fue evaluado por el área de infectología, que halló múltiples lesiones cavitadas en radiografía y tomografía computarizada (TC) de tórax previas (figura1), documentándose, asimismo, bacteriemia por Pseudomonas aeruginosa. Tales hallazgos motivaron la sospecha de endocarditis infecciosa, por lo cual se realizó un ecocardiograma transesofágico que mostró vegetación muy móvil y de pedículo estrecho de $12 \mathrm{~mm}$ de diámetro mayor, adherida a la superficie ventricular de la válvula pulmonar (figura 1), la cual no representaba anomalía funcional alguna.

Se inició manejo con meropenem a dosis de 2 gramos intravenosos cada 8 lioras, según patrón de sensibilidad, terapia que se prolongó durante las siguientes seis semanas, obteniendo durante el período de tratamiento y después de éste, hemocultivos negativos y ecocardiograma transesofágico sin evidencia de vegetaciones.

\section{Discusión}

Las primeras publicaciones sobre endocarditis infecciosa datan de 1885 , cuando se describió como una entidad de difícil diagnóstico y con una letalidad cercana al $100 \%$. Su presentación clínica es variada 


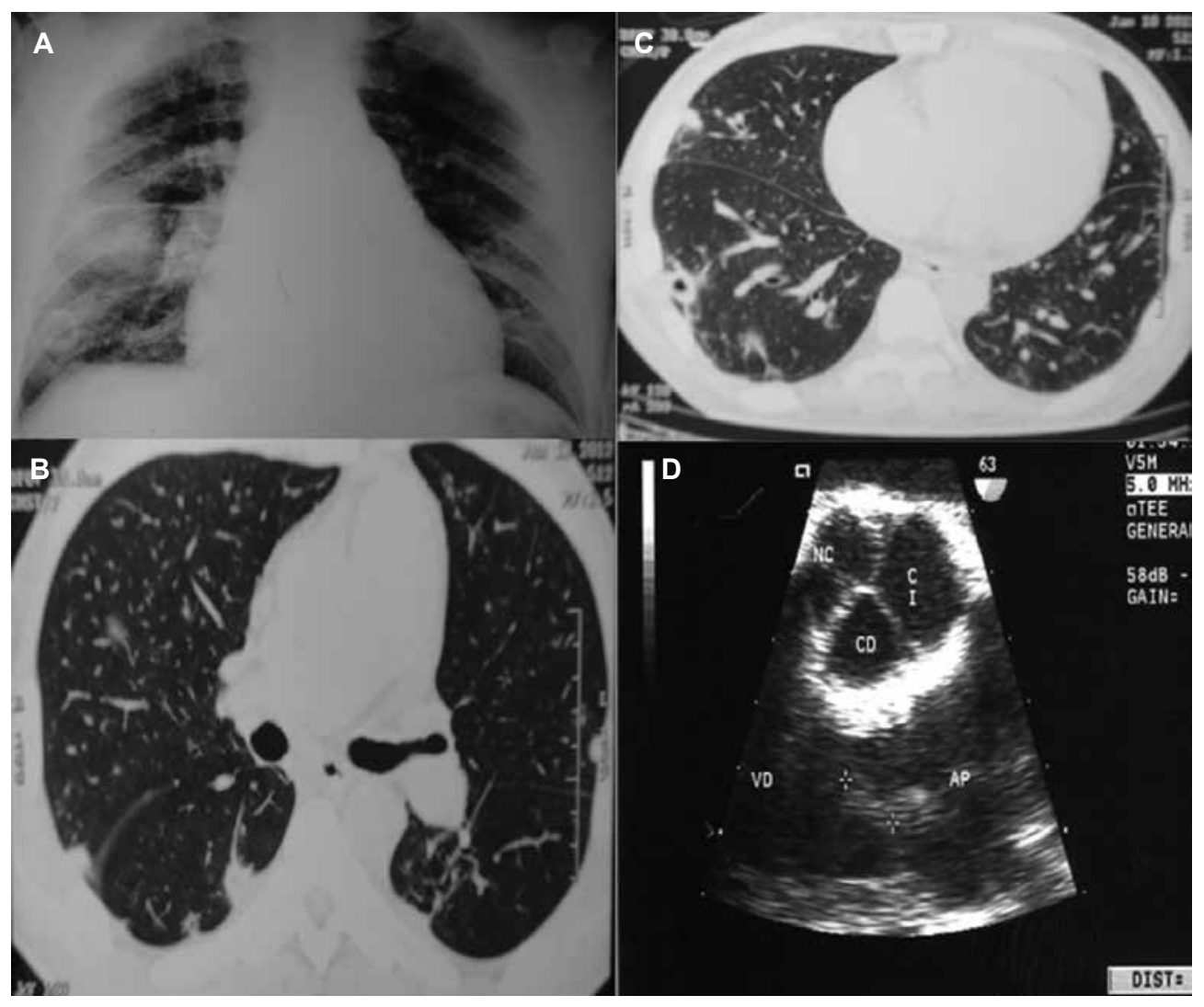

Figura 1. Imágenes de embolia séptica pulmonar. A. Múltiples nódulos a nivel periférico, incluso con cavidad en 1/3 inferior de hemitórax derecho. B. Nódulos periféricos en hemitórax derecho en los cuales se insinúa el signo del vaso nutricio C.Lesiones cavitadas periféricas en TAC de tórax. D. Imagen de vegetación en ecocardiograma transesofágico.

e inespecífica (7). Entre los factores de riesgo que predisponen al desarrollo de endocarditis infecciosa, se incluyen ISA, género femenino, válvulas cardiacas protésicas, enfermedad cardiaca estructural o historia de endocarditis infecciosa previa. Algunos estudios han demostrado tasas más altas de endocarditis infecciosa en pacientes con ISA infectados con el VIH que entre aquellos VIH negativos, y una relación inversa entre la ocurrencia de endocarditis infecciosa y el conteo de linfocitos CD4 $(4,8)$.

La introducción de la terapia antirretroviral (TARV) ha reducido la morbilidad, la mortalidad y las hospitalizaciones asociadas con la infección por VIH. Estudios en la era pre-TARV y en la etapa temprana de inicio de la misma, indicaron un aumento del riesgo de endocarditis infecciosa entre aquellos pacientes infectados con VIH, con mayores tasas de mortalidad en aquellos con inmunosupresión severa (8).

La incidencia de endocarditis infecciosa por gérmenes gramnegativos, permanece baja; sin embargo, la epidemiología y los factores de riesgo están cambiando. En un estudio realizado en 2007, los autores concluyeron que la endocarditis infecciosa debido a organismos gramnegativos no HACEK está mucho más asociada con dispositivos intravasculares implantados que con el uso de drogas intravenosas. Diversas revisiones han demostrado el rol de las infecciones asociadas con el cuidado de la salud en el aumento de la prevalencia de la endocarditis infecciosa por patógenos gramnegativos. Un análisis multicéntrico de 2.761 pacientes con endocarditis infecciosa de un total de 61 hospitales, encontró que los organismos no HACEK son responsables de 
menos del 2\% de los casos. Entre los pacientes con infecciones no HACEK, 59\% tuvieron dispositivos implantables endovasculares y válvulas protésicas, pero sólo 4\% fueron ISA. Más de la mitad de los pacientes con infecciones por estos gérmenes requirieron intervención quirúrgica y $24 \%$ murieron. En ese mismo estudio, los patógenos más comunes en pacientes con endocarditis infecciosa por bacilos gramnegativos no HACEK, fueron Escherichia coli (29\%) y Pseudomonas aeruginosa (22\%) $(4,5)$.

La incidencia de endocarditis infecciosa en pacientes VIH positivos, fue calculada en un $2 \%$ en un estudio multicéntrico retrospectivo (Francia, España, Estados Unidos) (2) en 647 pacientes no usuarios de drogas intravenosas. Grinberg y colaboradores comprobaron que los pacientes infectados por el VIH tipo 1, tienen un riesgo cuarenta veces mayor de desarrollar una endocarditis infecciosa en comparación con los no infectados por el retrovirus, hecho que para aquel entonces estaba influenciado notablemente por el uso de drogas intravenosas (9).

En un estudio publicado en 2006, Kelly y colaboradores determinaron la incidencia de endocarditis infecciosa entre 1990-1995, siendo de $20,5 \times 1000$ personas año, mientras en el periodo comprendido entre 1996-2002 la incidencia calculada fue de 6,6 x 1000 personas año. La mayoría de los casos de endocarditis infecciosa correspondió a afroamericanos $(89,7 \%)$, hombres $(65,5 \%)$, con una media de edad de 40 años. Los principales factores de riesgo identificados en los pacientes VIH positivos, fueron: ISA $(84,5 \%)$, transmisión heterosexual (31\%) y homosexualidad $(13,8 \%)$. La media de CD4 al momento de la endocarditis infecciosa fue de 68 células $/ \mathrm{mm}^{3}$, con una media de carga viral de 78.288 copias $/ \mathrm{ml} ; 75 \%$ de los pacientes tuvieron infección concomitante con virus de la hepatitis $\mathrm{C}$. En el tiempo del diagnóstico, 31\% de los pacientes estuvieron en TARV. En un análisis multivariado posterior, los casos fueron más probablemente usuarios de drogas intravenosas, y valores más bajos de CD4 (menos de 50 células $/ \mathrm{mm}^{3}$ ) y mayor carga viral (más de 100.000 copias) (8).

Como conclusiones de éste y otros análisis se considera que la endocarditis infecciosa es un diagnóstico común en pacientes infectados con VIH
$(6,6 \times 1,000$ personas año vs. $1 \times 1.000$ persona año en la población general). Adicionalmente, en este mismo estudio se indica que la endocarditis infecciosa en un paciente con VIH entraña mayor riesgo de recurrencia y mortalidad a un año (16-44\% con el mismo microorganismo- y $52 \%$, respectivamente). A partir de los resultados del estudio en cuestión se lanza la hipótesis que, si bien la TARV no se asocia con disminución del riesgo de bacteriemia, si podría reducir la posibilidad de que un organismo cause endocarditis (8).

La mortalidad global de la endocarditis infecciosa en personas con ISA es de alrededor del 10\%; en la serie de Hechtet y colaboradores, que incluyó sólo pacientes con endocarditis infecciosa de localización derecha, fue del 7\% y en la serie de pacientes argentinos publicada en 2004 alcanzó el 10,9\% (9). La infección por el VIH con inmunodeficiencia grave asociada (recuento de CD4 menor de 200 cél/ $\mu \mathrm{L}$ ), vegetaciones de más de $2 \mathrm{~cm}$ y desarrollo del síndrome de dificultad respiratoria del adulto o de shock séptico, son algunos de los factores que ensombrecen el pronóstico de la endocarditis infecciosa en este grupo de pacientes (9). En este caso, pese a un conteo de CD4 menor de 200, la respuesta a la terapia médica instaurada fue satisfactoria.

La endocarditis infecciosa por Pseudomonas aeruginosa es una entidad poco frecuente, de difícil diagnóstico y alta mortalidad. Se ha descrito principalmente en personas con ISA, con compromiso de las válvulas tricúspide y pulmonar. Las vegetaciones detectadas por la ecocardiografía comprometen la válvula tricúspide en más del $80 \%$ de los casos de acuerdo con las distintas series. No se observa una diferencia significativa en la sensibilidad y especificidad entre el ecocardiograma transesofágico y transtorácico, a diferencia de lo que ocurre en las válvulas mitral y aórtica. El compromiso bilateral es raro (10-12).

Cuando se evalúa un paciente con VIH y se encuentran cavidades a nivel pulmonar dentro de los estudios imaginológicos, es indispensable diferenciar el cuadro entre aquellos de presentación aguda/subaguda y aquellos de presentación crónica. Entre los primeros, adquieren importancia las infecciones por M. tuberculosis, S. aureus, anerobios, 
legionellosis y Pseudomonas aeruginosa. En el último grupo, se destacan: micobacterias, Nocardia, hongos y Rhodococcus equi. También es importante tener en consideración que las lesiones cavitadas pueden presentarse particularmente en el contexto de embolia séptica e infección por gérmenes gramnegativos, como en el paciente en cuestión, siendo la presencia del característico vaso nutricio en la tomografía de tórax, un hallazgo de importancia capital (13-15).

Las características clínicas de la embolización séptica pulmonar son inespecíficas, y el diagnóstico es retardado. Los cultivos de sangre, la tomografía de tórax y la ecocardiografía son invaluables en su estudio. Los hallazgos radiográficos típicos incluyen lesiones en parches del espacio aéreo simulando bronconeumonía, múltiples nódulos en forma de cuña o mal definidas, de tamaño variable, localizadas en la periferia y lesiones que abomban la pleura y están localizadas al final de los vasos en la tomografía computarizada (6).

Kwon y colaboradores (6) reportaron recientemente que el tamaño de los nódulos en embolia séptica por grampositivos fue mucho mayor que la observada en gramnegativos, así como fueron más frecuentes las cavidades y el broncograma aéreo, mientras un halo de atenuación en vidrio-esmerilado fue más común en embolia por gramnegativos.

En la endocarditis de cavidades derechas, la densidad de las bacterias en las vegetaciones es menor que en la de las cavidades izquierdas, lo cual aumenta las probabilidades de éxito terapéutico con el tratamiento médico, como sucedió con nuestro paciente. Este tipo de endocarditis presenta una buena respuesta al manejo antibiótico, a diferencia de la endocarditis de cavidades izquierdas con compromiso de las válvulas aórtica y mitral, cuyo tratamiento de elección es el reemplazo valvular, dada la alta tasa de mortalidad descrita con el tratamiento médico (16-18).

En conclusión, podría decirse que la endocarditis infecciosa con compromiso del corazón derecho es una entidad más frecuente en los pacientes con infección por el virus de la inmunodeficiencia adquirida que en la población general y que, independientemente del uso o abuso de drogas intravenosas, esta puede ser originada por gérmenes gramnegativos, entre ellos por Pseudomonas aeruginosa, principalmente en aquellos pacientes con estancia hospitalaria prolongada, bacteriemias nosocomiales o portadores de catéteres venosos centrales para su manejo, no siendo rara su asociación con embolia séptica pulmonar. Pese a que existen pocos casos en la literatura, el manejo médico farmacológico parece ser una opción para este grupo de pacientes.

\section{Conflictos de intereses}

Los autores declaran no tener conflicto de intereses.

\section{Agradecimientos}

Al Doctor Carlos Ignacio Gómez Roldán, Médico internista e Infectólogo del Hospital Pablo Tobón Uribe de Medellín-Colombia.

\section{Bibliografía}

1. Taylor J. The Guidelines for management of infective endocarditis reviewed. Eur Heart J. 2009;30(19):2185-6.

2. Miró JM, del Río A, Mestres CA. Infective endocarditis and cardiac surgery in intravenous drug abusers and HIV-1 infected patients. CardiolClin. 2003;21(2):167-84.

3. Nishimura RA, Carabello BA, Faxon DP, Freed MD, Lytle BW, O'Gara PT. ACC/AHA 2008 guideline update on valvular heart disease focused update on infective endocarditis: a report of the American College of Cardiology/American Heart Association Task Force on Practice Guidelines endorsed by the Society of Cardiovascular Anesthesiologists, Society for Cardiovascular Angiography and Interventions, and Society of Thoracic Surgeons. Catheter CardiovascInterv. 2008;72(3):E1-12.

4. Reyes MP, Ali A, Mendes RE, Biedenbach DJ. Resurgence of pseudomonas endocarditis in Detroit, 2006-2008.Medicine (Baltimore).2009;88(5):294-301.

5. Morpeth S, Murdoch D, Cabell CH, Karchmer AW, Pappas P, Levine D. Non-HACEK gram-negative bacillus endocarditis. Ann Intern Med. 2007;147(12):829-35.

6. Jorens PG, Van Marck E, Snoeckx A, Parizel PM. Nonthrombotic pulmonary embolism.EurRespir J. 2009;34(2):452-74.

7. Ordóñez KM, Hernández OA, Cortés JA, López MJ, Alfonso G, Junca A. Endocarditis infecciosa izquierda por Pseudomonas aeruginosa tratada médicamente.Biomédica.2010; 30:164-9. 
8. Gebo KA, Burkey MD, Lucas GM, Moore RD, Wilson LE. Incidence of, risk factors for, clinical presentation, and 1-year outcomes of infective endocarditis in an urban HIV cohort.J AcquirImmuneDeficSyndr. 2006;43(4):426-32.

9. Corti ME, Palmieri OJ, Villafañe MF, Trione N. Evaluación de 61 episodios de endocarditis infecciosa adictos a drogas intravenosas e infección por el virus de la inmunodeficiencia tipo-1. RevArgMicrobiol.2004;36:85-7.

10. Venkatesan A, Spalding C, Speedie A, Sinha G, Rumbaugh JA. Pseudomonas aeruginosa infective endocarditis presenting as bacterial meningitis.J Infect. 2005;51(4):e199-202.

11. Dawson NL, Brumble LM, Pritt BS, Yao JD, Echols JD, Alvarez S. Left-sided Pseudomonas aeruginosa endocarditis in patients without injection drug use.Medicine (Baltimore). 2011;90(4):250-5.

12. Navarrete Navarrete N, Tapia Gómez A, López-Gómez M, López-Ruz MA, Jiménez Alonso J. Endocarditis por Pseudomonas aeruginosa. Presentación de un caso atípico y revisión de la literatura. AnMedInt (Madrid). 2007;24(2):99-100.

13. Hull MW, Phillips P, Montaner JS. Changing global epidemiology of pulmonary manifestations of HIV/AIDS. Chest. 2008;134(6):1287-98.

14. Changing global epidemiology of pulmonary manifestations of HIV/AIDS. Chest. 2008;134(6):1287-98.

15. Allen $\mathrm{CM}, \mathrm{Al}-\mathrm{Jahdali} \mathrm{HH}$, Irion $\mathrm{KL}, \mathrm{Al}$ Ghanem $\mathrm{S}$, Gouda $\mathrm{A}$, Khan AN. Imaging lung manifestations of HIV/AIDS.Ann Thorac Med. 2010;5(4):201-16.

16. Dodd JD, Souza CA, Müller NL. High-resolution MDCT of pulmonary septic embolism: evaluation of the feeding vessel sign.AJR Am J Roentgenol. 2006;187(3):623-9.

17. Yasar KK, Pehlivanoglu F, Gursoy S, Sengoz G. Tricuspid endocarditis and septic pulmonary embolism in an intravenous drug user with advanced HIV infection. Oman Med J. 2011;26(5):365-7.

18. Tembe AG, Kharbanda P, Dalal JJ, Vaishnav G, Joshi VR.Infective endocarditis--a tale of two cases and the lessons (re)learned.J Assoc Physicians India. 2010;58:319-22.

19. McDonald JR. Acute infective endocarditis.Infect Dis Clin North Am. 2009;23(3):643-64. 